مد النتشار الغالى الكهروغنلليسي

\author{
مؤبد عزبز العبيهي

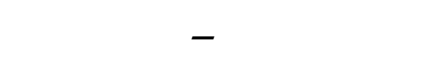 \\ جلمعة الموط
}

(2002/3/9 2002/8/1 ، تاريخ الإيخ القبول)

\title{
الملهص
}

هذف البحث إلى تحديد مدى انتشار الفاعل الكهرومغنطيسي. وقد م ذلك بالاعتماد على ظاريـة

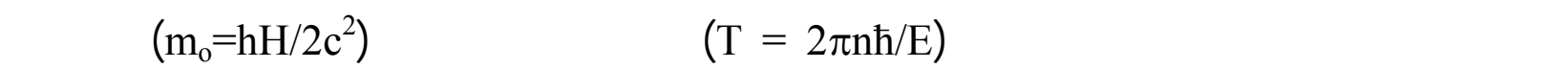

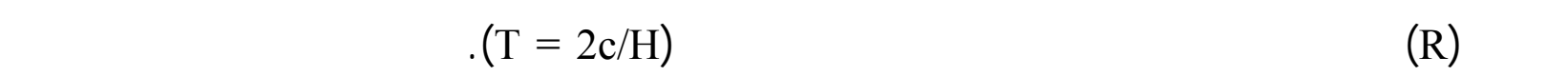
آخرون قبلنا.

\section{The Range of Distribution of Electromagnetic Reaction}

\author{
Muayad A. Al-Obaydi \\ Department of Physics \\ College of Science \\ Mosul University
}

\begin{abstract}
This research aimed to find the rang of the electromagnetic reaction. It does that according to the theory of secondary photons, quantized time $(\mathrm{T}=2 \pi \mathrm{nh} / \mathrm{E})$, and the photon rest mass $\left(m_{0}=h H / 2 c^{2}\right)$, and by calculating the range of the photon at this time its found that it is $(\mathrm{T}=2 \mathrm{c} / \mathrm{H})$, and it is with full agreement with the result that found previously
\end{abstract}

\section{المقمة}

في الاليكتروداينمك الكم (QED) يم التعلل مع الفوتون، باعتباره جسيما لا يمتلك كتلةس كون وذا عمر لا نهائي، ومن مُ فان مدى النتشار القاعل الكهرومغنلطيسي (Rh

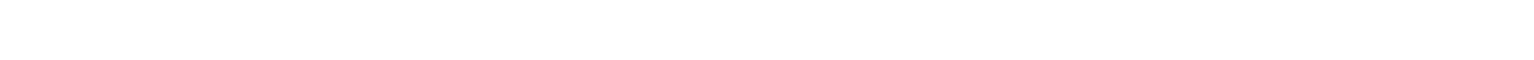
التعليم، بينما حققته اعقد من ذلك (Strnad, 1986) ويرى اينشتاين (Einstein) ان الفوتون مفهوه غي -ر 
مفهوم-على الرغم من النه لستعمله في تفسيرهظاهرة التأثير الكهروضوئي-إذ يقول (ان خمسنسنة من التألمل لم تجعلني قريبا من لجابة للسؤل : ما هو كم الضوء)، (Speziali, 1972).

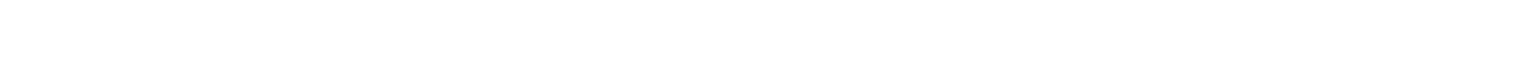

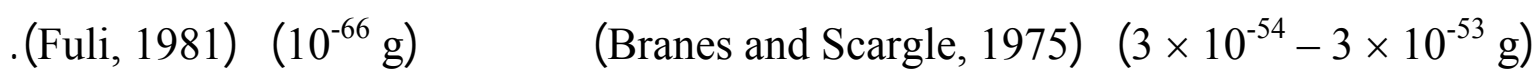

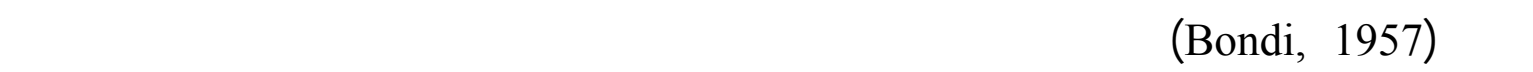
ثلاثة النواع هي الكتلة القصورية (Inertial mass) والكتل ـة الجنبي ـة الفاعل ة (Active gravitational) والكتلة الجنبية المفعلة (Passive gravitational).

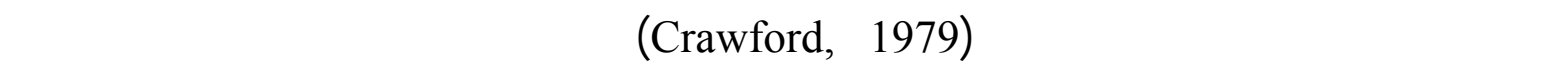

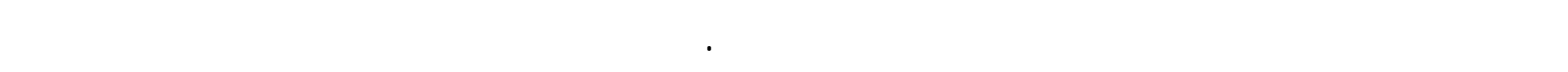

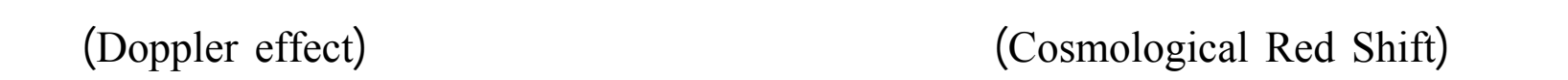

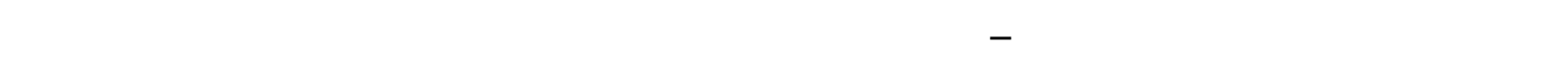

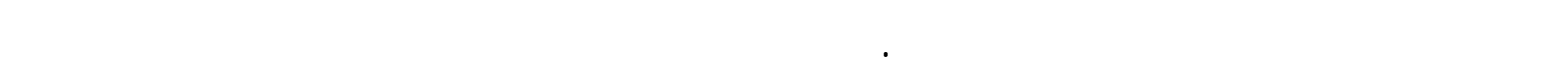
جدية ـ ان هذه البحوث وما طرحهه من تصورات ونتائج تخص كتلةسكون الفوتون وعمره، تذفع باتجها

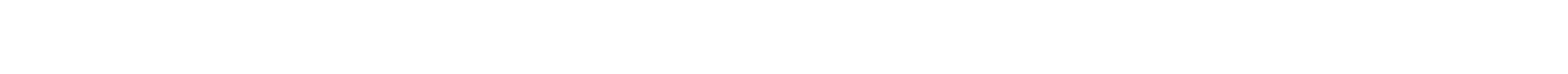

$$
\text { . }\left(\mathrm{R}_{\mathrm{ph}}=\mathrm{cT} \mathrm{T}_{\mathrm{ph}}\right) \mathrm{T}_{\mathrm{ph}}
$$

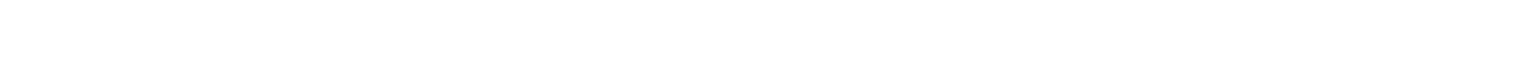

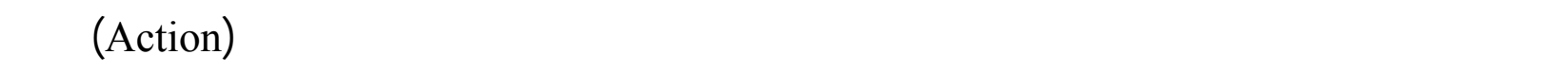

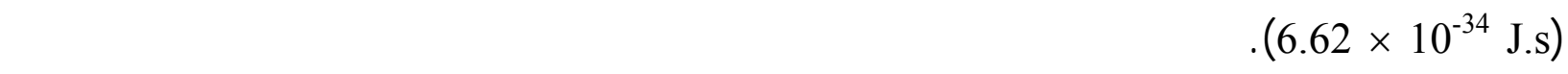
(3) $\left.310^{8} \mathrm{~m} / \mathrm{s}\right)$

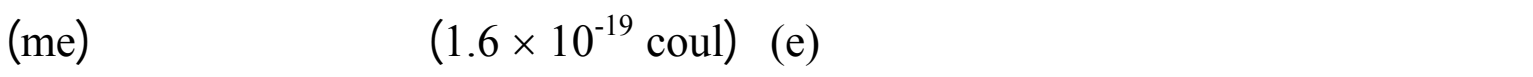

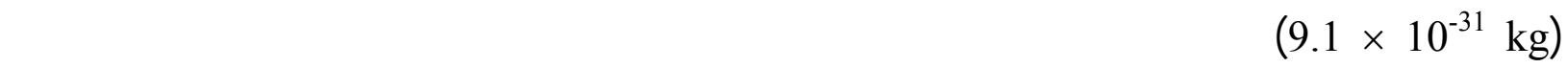
الثوالبت الكونية ووضع وحدات قيلس × × الثبعية تعتمد هذه المحددات أو الثوابت الكونية (Dirac, 1937).

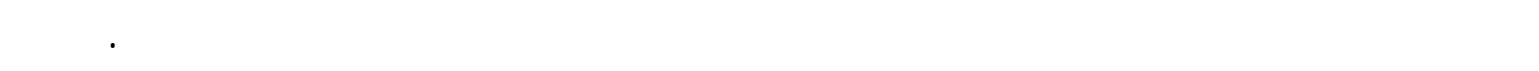

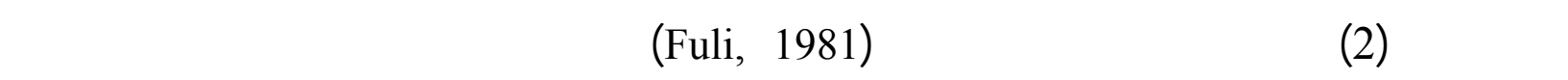

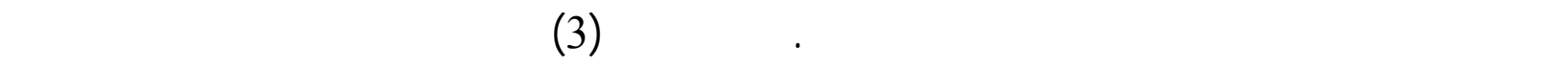

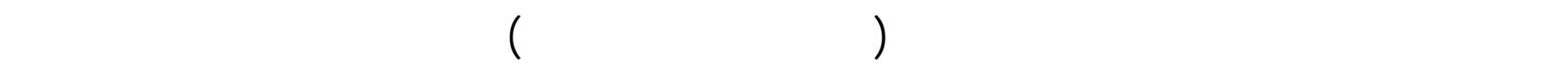
الفوتون. ونوقثت النتائج في الفقرة (4). 


\section{كتلفسكون الفوتهن:}

اذا ما اعتمدنا ظارية الانبعلث الثانوي الناتج عن تقاعل الفوتون مع المجل الج ذبي ، ف ـان هـ ذا

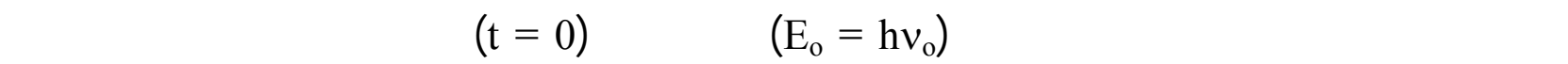

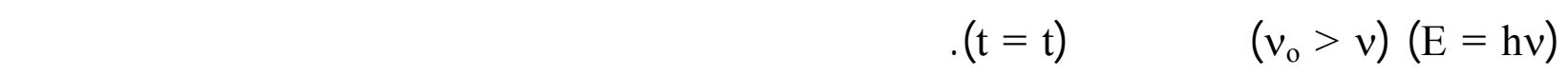

$$
\partial \mathrm{h} v / \partial \mathrm{t}=\mathrm{h} v_{\mathrm{o}} / \mathrm{t}_{\mathrm{c}}
$$$$
\text { في الطلةة والزمن (Fuli, 1981). }
$$

$$
\mathrm{h} v=\mathrm{h} v_{\mathrm{o}} \exp \left[-\mathrm{r} / \mathrm{r}_{\mathrm{c}}\right]
$$$$
\text { التي يمكن ان نحطل منها على }
$$

حيث

سكون الفوتون.

$$
\text { من معادلة (2) يمكن الحصول على معلمل الانحرف الاحمر (Z) : }
$$

$$
\mathrm{Z}=\frac{v_{0}-v}{v}=\mathrm{e}^{\mathrm{r} / \mathrm{r}_{\mathrm{c}}}-1
$$

$\mathrm{Z}=2 \mathrm{mcr} / \mathrm{h}$

$$
\text { عند }
$$

$$
\begin{aligned}
& \mathrm{Z}=\mathrm{Hr} / \mathrm{c} \\
& \mathrm{m}=\mathrm{hH} / 2 \mathrm{c}^{2} \\
& \text { حيث (H) ثالمت هابل . من معادلة (5) و (4) نحطل على : } \\
& \text { وحيث لن (Z) بهسب علاقة هالبل هو : }
\end{aligned}
$$

والتي هي كتلةسكون الفوتون .

\section{تحسيدمى النتشار الفالل الكهومغنلاليسي:}

لكل جسيم نسبوي كم زمني الصغر لا يمكن متابعة الطور الزمني للج سيم بع عاده (Misra, 1995)

والقد وجنا في عطلسابق (Al-Obaydi, 2001) ان كم الزمن الخاص بالفوتون هو : $\mathrm{T}=2 \pi \mathrm{n} \hbar / \mathrm{E}$

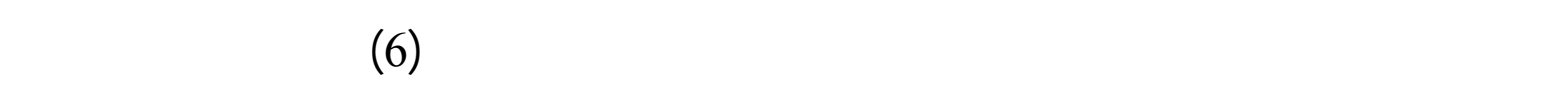

$$
\mathrm{T}=2 / \mathrm{H}
$$

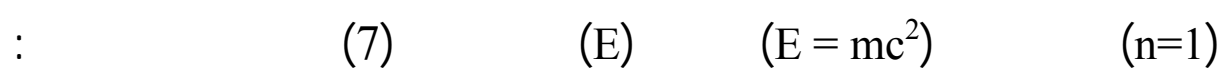

وحيث ان (T) هي عمر الفوتون ف فانحسيكون بالامكان هسب المدى الذيسيطعه الفوتون في هذا

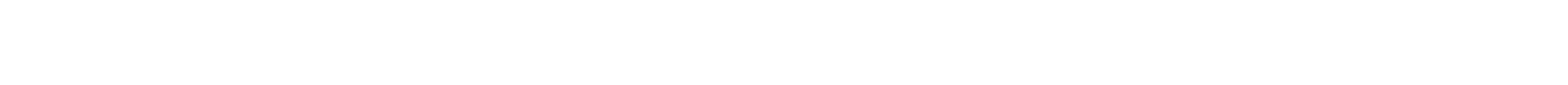
المدى (R) 
$\mathrm{R}_{\mathrm{ph}}=4 \times 10^{10} \mathrm{Ly}$

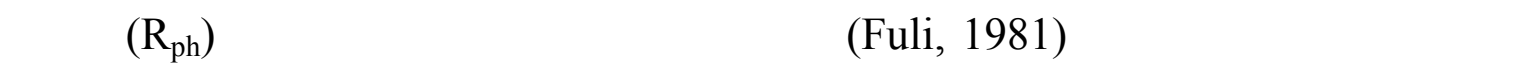

( مهي نتيجة مختلفة عن التي توصلنا اليها وسنناقشها في فقرة النتئج و المناقثة . (Rh $=2$. $10^{9}$ Ly)

\section{النتائج والمنلثشة}

تشير النتيجة الاسلسية للبحث ان مدى انتثار الفاعل الكهرومغنلطيسي ليس لانهائيا بل هوم دمى

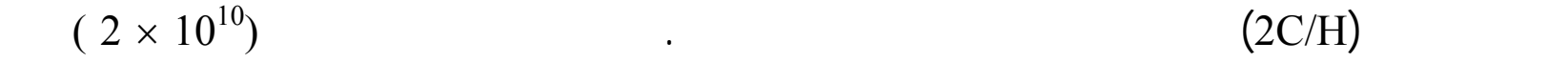

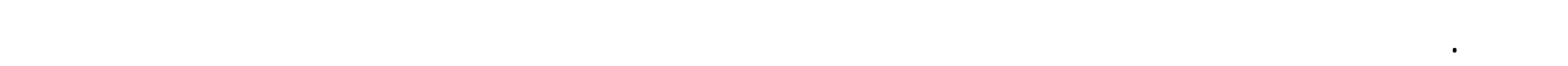

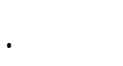

وحيث ان النتيجة تعتمد ثابت هالب فانه لا يمكن الجزم بان هذه التتيجة نهائية. لان ثابت هابل الذي يعتمد عليه المدى ليس محددا تحديدا نهائيا (Weinberg, 1972). ومما تشير اليه النتيجة ان تكميم الزمن يمكن طبيقه في البحوث الكونية إذ تبين في الفق ـرة (3) لن النتائج التي م التوصل اليها باعتماد الانحرف الاحمر يمكن الوصول اليها بلستخدلم تكميم الزمن. وم ن مُ يعد البهث طبيقامن طبيقت تكميم الزهن. ان المقدار الذي توصلنا اليه يختلف من ذاك الذي وجده (Fuli) على الرغم مه ن تماثة لـ ال ـصيغة

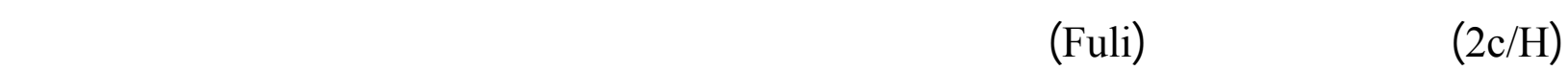

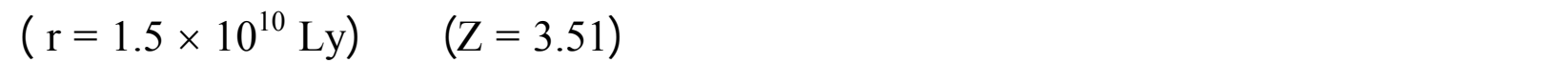
وقد لا نجد تقسيرا لنتائجه الرقمية إلا في لنه لستخدم مقدارا لثلت هالبل غير الذي لستخدمنه.

\section{المصار لأجنبية}

Al-Obaydi, M.A., 2001.Time Quantization,Unpublished Ph.D.Thesis, Mosul Univ. Bondi, H., 1957. Negative mass in general relativity, Rev. Mod. Phys., 29(3): 423 - 428. Branes, A. and Scargle, J.D., 1975. Improved Upper limit on photon rest mass,Phys. Rev. lett. 35(17) : $1117-1120$.

Crawford, D.F., 1979. Photon decay in curved space-time, Nature, 277: 633-635.

Dirac, F.A.M., 1937. The Cosmological constants, Nature, Feb.: 323.

Fuli, Li.,1981.An Estimate of the photon rest mass, lett. Nuvo Cimento 31(8):289 - 296. Misra, B., 1995. Form time operators to chronons, Foundation of Phys. 25(7): 1087-1104.

Speziali, P., 1972. Ed, Albert Einstein - Michele Besso Correspodence 1903-1955, Hermann, Paris.

Strnad, J., 1986. Photone in introductory quantum physics, Am. J. Phy. 54(7): 650-652.

Weinberg, S., 1972. Growitation and Cosmology, John Wielly and Sons Inc. Canada. pp.441-450. 